\title{
How short are ultra short light pulses? (looking back to the mid sixties)
}

\author{
H. P. Weber \\ heinz.weber@iap.unibe.ch \\ R. Dändliker \\ rene.dandliker@epfl.ch \\ Institute of Applied Physics, University of Bern, CH-3012 Bern, Switzerland \\ Institute of Microengineering. EPFL, CH-1015 Lausanne, Switzerland
}

With the arrival of mode locking for Q-switched lasers to generate ultra short light pulses, a method to measure their expected time duration in the psec range was needed. A novel method, based on an intensity correlation measurement using optical second harmonic generation, was developed. Other reported approaches for the same purpose were critically analysed. Theoretical and subsequent experimental studies lead to surprising new insight into the ultra fast temporal behaviour of broadband laser radiation: any non mode locked multimode emission of a laser consists of random intensity fluctuations with duration of the total inverse band width of emitted radiation. However, it was shown, that with mode locking isolated ultra short pulses of psec duration can be generated. This article summarizes activities performed in the mid sixties at the University of Berne, Switzerland. [DOI: 10.2971/jeos.2010.10050S]

Keywords: ultra short light pulses, mode locking, intensity correlation, nonlinear optics

\section{INTRODUCTION}

How short are ultra short light pulses? This was a key question in the mid sixties of the last century. A novel approach to generate extremely short optical pulses was the method of locking the phases of oscillating modes in a laser. A clever approach to realize the necessary internal modulation of light within the laser resonator was the employment of a fast recovering saturable absorber. DeMaria et al. [1] generated by this method trains of ultra short pulses with Nd:glass as the laser active material. He suggested that the individual pulses might be as short as picoseconds $\left(10^{-12} \mathrm{sec}\right)$, the inverse of the total oscillating bandwidth.

With this type of experiments he opened up an entirely new range of timescales of investigations. At that time the fastest time duration of optical pulses as well as the time response of optical detectors were both in the range of $1 \mathrm{nsec}$. Thus, detectors were about 1000 times too slow. In addition, fundamental questions on optical instrumentation came up; is traditional equipment still performing the same manner in this time range? Optical instruments like spectrometers and Fabry-Perrots have dimensions of at least several $\mathrm{cm}$ in length, but psec wave packets are only $0.3 \mathrm{~mm}$ in length.

\section{MEASURING SHORT PULSES USING NONLINEAR OPTICAL EFFECTS}

The first fundamental question was: What can we tell about the real time duration of the pulses of mode locked lasers. Approaches to obtain information from any interferometric measurements fail, because they are only alternatives to a measurement (Fourier-transforms) of the totally emitted bandwidth. Photo detectors and electronic signals were too slow to resolve these time scales; thus it was realized, that it had to be the light pulse itself to act as time marker. Such an interaction can only be accomplished with a nonlinear optical process. The interaction had to be an interaction of the light pulse with a duplicate of itself. A process of nonlinear optical interaction fulfilling this requirement had been found previously in the form of type II phase matching [2]: Propagation directions in optically nonlinear crystals were found, where two light beams of equal wavelength but of orthogonal polarisations generated phase matched second harmonic radiation only when both signals were present at the same time. A beam of either polarisation by itself generated no second harmonic output. The suggested approach to measure time duration [3] was a Michelson-like arrangement (Figure 1). The 45 degree polarised radiation entering the arrangement was in the two arms polarised by the two orthogonally polarising GlanThompson prisms and recombined, entering the properly oriented nonlinear crystal. Background free operation could easily be proved by blocking one of the arms. By changing the length of one of the arms of the Michelson a relative delay $\Delta l$ between the two radiations could be established. The time averaged intensity of the resulting second harmonic corresponds to the intensity autocorrelation of the ultra short light pulse. The necessary relative delay to make the second harmonic signal to disappear determined the duration of the pulses. The results of such a measurement (Figure 2) showed that ideally isolated ultra short pulses were generated [4]. The pulses were as short as 12.5 psec, however, not as short as 3 psec, which the spectral width of the radiation would have permitted. Experiments at that times with mode locked pulses were rather time consuming. Experiments with pulsed lasers and liquid saturable absorbers allowed only repetition rates from shot to shot of typically 1 minute and sampling at different delays $\Delta l$ 


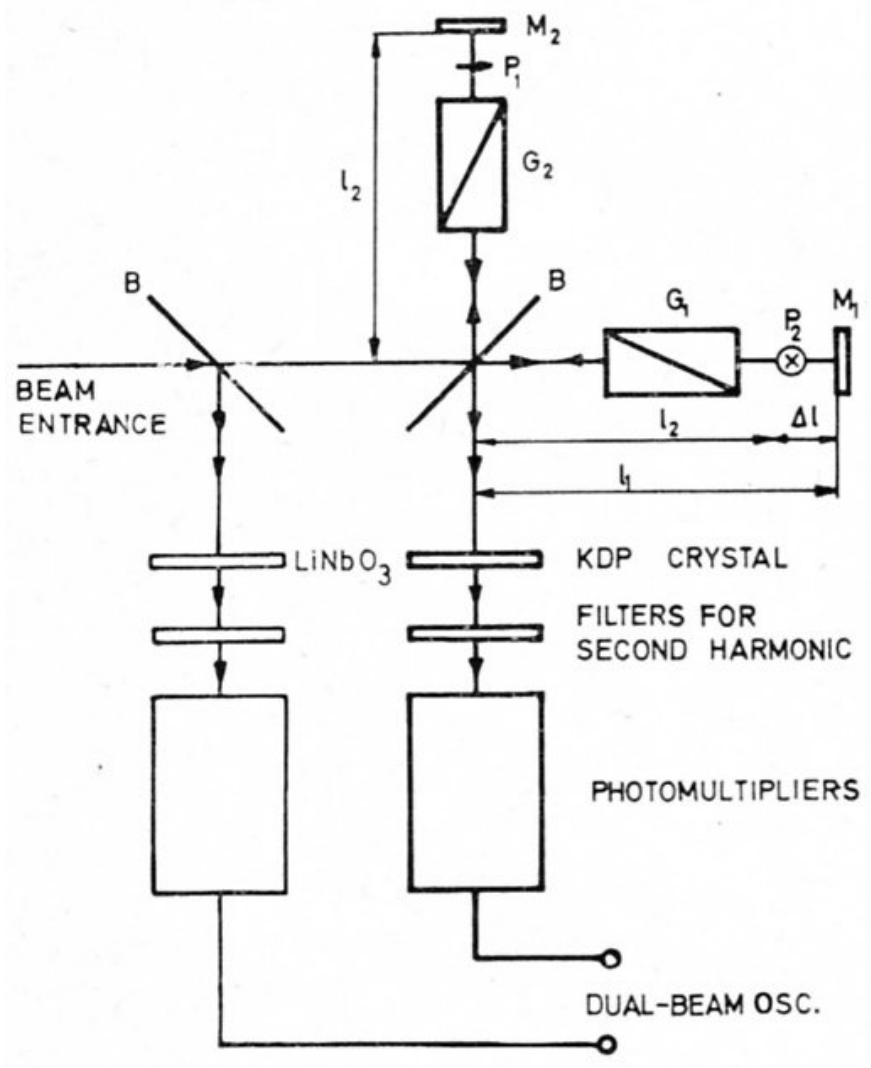

FIG. 1 Experimental arrangement for measuring the autocorrelation function of ultra short pulses [4]. $M_{1,2}$ mirrors, $G_{1,2}$ Glan Thompson prisms, polarising according to the directions $\mathrm{P}_{1,2}$. The second harmonic generated by the $\mathrm{LiNbO}_{3}$ crystal served as a reference to control shot to shot variations.

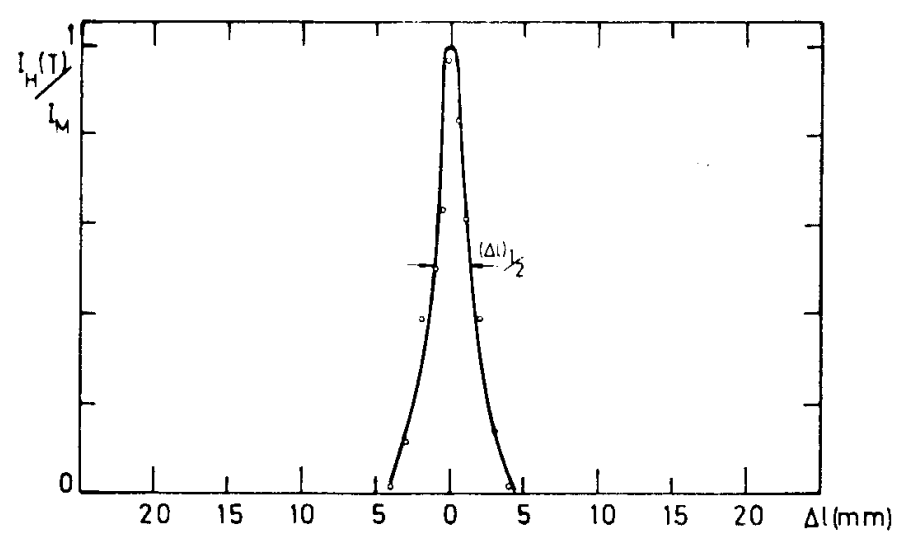

FIG. 2 Measured autocorrelation function from a Q-switched mode locked pulse train [4].

was needed to record a complete autocorrelation. Each measured point corresponds to an integration over the entire pulse train of the Q-switched laser emission.

An interesting alternative to second harmonic generation was thus to use two-photon absorption induced fluorescence as nonlinear process. Thereby, the initial beam is split into two parts that enter counter propagating a cell filled with the fluorescing dye. A straightforward way to accomplish this was to employ a reflecting mirror at the end of the cell to generate the second beam (Figure 3) [5]. The fluorescence can be recorded photographically, showing directly the enhanced interval of the overlapping pulses. Here, only one shot is needed

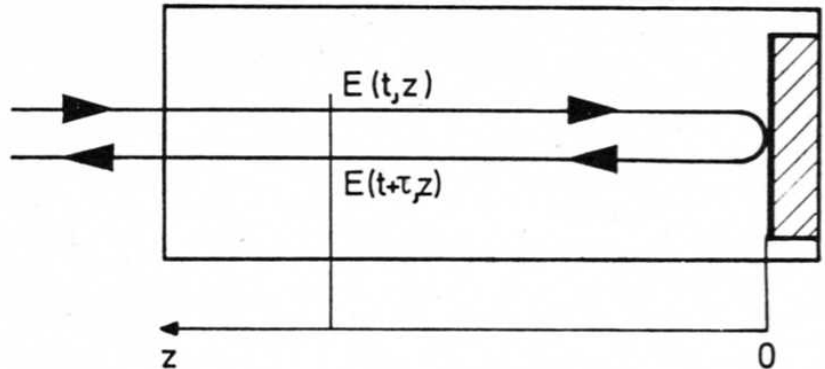

FIG. 3 Diagram of the experimental arrangement for pulse width measurements by two-photon excitation of fluorescence [7].

per measurement. The drawback is however, that in contrast to the above second harmonic generation the overlap is superimposed on a background generated by the fluorescence of each beam by itself $[5,6]$. The peak of the signal is ideally 3 times the background signal. The experimentally determined contrast, which is difficult to be measured accurately, did reach a much lower value, but the time durations evaluated by this method from the length of the enhanced fluorescence were ideally short, i.e. transform limited as given by the total bandwidth of the radiation. Puzzled by this result a detailed study seemed to be appropriate [6, 7]. The corresponding results will be presented and discussed in the following chapters.

\section{ANALYSES OF MEASURING METHODS}

Both methods attempt to measure the intensity correlation functions of the radiation fields. For calculation we use the analytic signals $V(t)$ associated to the real electric field $E(t)$. The intensities obey the well known relation

$$
I(t)=V(t) \cdot V^{*}(t),
$$

where $V^{*}(t)$ is the conjugate complex of $V(t)$. The signal with the relative delay $\tau$ is described by $V(t+\tau)$. In the measurement using second harmonic generation $(\mathrm{SH}) \tau$ is given by the relative displacement of the arms of the Michelson. In the case of the two-photon absorption induced fluorescence (TPA) the observed structure is a local display of the delay $\tau$, where the position of the mirror corresponds to $\tau$ equal zero.

\subsection{Second harmonic generation}

The time averaged intensity of the second harmonic signal $S(\tau)$ generated by type II phase matching becomes

$$
S(\tau) \propto\left\langle V(t) \cdot V(t+\tau) \cdot V^{*}(t) \cdot V^{*}(t+\tau)\right\rangle .
$$

This signal, normalized to the second harmonic without delay (see Figure 1), is exactly the intensity correlation function $G^{2}(\tau)$. Using the abbreviations $V=V(t)$ and $V_{\tau}=V(t+\tau)$, we obtain

$$
G^{2}(\tau)=\frac{\left\langle V \cdot V_{\tau} \cdot V^{*} \cdot V_{\tau}^{*}\right\rangle}{\left\langle V \cdot V \cdot V^{*} \cdot V^{*}\right\rangle}
$$

or expressed in the more familiar form

$$
G^{2}(\tau)=(\bar{I})^{-2} \int_{-\infty}^{\infty} I(t) \cdot I(t+\tau) \cdot d t
$$


where $I(t)$ is the instantaneous intensity and $\bar{I}(t)$ is the mean intensity. The signal at the second harmonic thus corresponds exactly to

$$
S(\tau)=G^{2}(\tau)
$$

For ideal mode locking and a delay $\tau$ which is large compared with the inverse bandwidth $(\Delta v)^{-1}$, the value of the correlation function $G^{2}\left(\tau \gg(\Delta v)^{-1}\right)$ goes to zero. The contrast $C_{i d}^{S H}$ from the maximum to the minimum of $G^{2}(\tau)$ becomes thus

$$
C_{i d}^{S H}=\frac{G^{2}(0)}{G^{2}\left(\tau \gg(\Delta v)^{-1}\right)}=\infty .
$$

The width of $G^{2}(\tau)$ is a direct measure of the actual time duration of the short pulse.

\subsection{Two-photon absorption induced fluorescence}

The observed fluorescence intensity is calculated from $V(t)+$ $V(t+\tau)$, the superposition of the two signals. The delay $\tau$ is given by the position $z$ in the absorption cell (Figure 3). Using the abbreviations $V=V(t)$ and $V_{\tau}=V(t+\tau)$ the time averaged fluorescence intensity $F(\tau)$ becomes

$$
\begin{aligned}
F(\tau) \propto\langle(V+ & \left.\left.V_{\tau}\right) \cdot\left(V+V_{\tau}\right) \cdot\left(V^{*}+V_{\tau}^{*}\right) \cdot\left(V^{*}+V_{\tau}^{*}\right)\right\rangle \\
=2\langle V & \left.V V^{*} V^{*}\right\rangle+4\left\langle V V_{\tau} V^{*} V_{\tau}^{*}\right\rangle \\
& +2\left\langle\left(V V^{*}+V_{\tau} V_{\tau}^{*}\right)\left(V V_{\tau}^{*}+V^{*} V_{\tau}\right)\right\rangle \\
& +\left\langle V V_{\tau}^{*} V V_{\tau}^{*}+V^{*} V_{\tau} V^{*} V_{\tau}\right\rangle
\end{aligned}
$$

The first two terms are again intensity correlation functions $G^{2}$, but now of the form $G^{2}(0)$ and $G^{2}(\tau)$, respectively. The last two terms are spatial oscillation with a period of half the wavelength, and thus not resolved by the observation; they average out to zero. The spatial structure of the time averaged fluorescence is therefore described by

$$
F(\tau) \propto G^{2}(0)+2 \cdot G^{2}(\tau)
$$

For an ideally short pulse the contrast $C_{i d}^{T P A}$ of the fluorescence trace from the maximum to the minimum must thus be

$$
C_{i d}^{T P A}=\frac{G^{2}(0)+2 \cdot G^{2}(0)}{G^{2}(0)+2 \cdot G^{2}\left(\tau \gg(\Delta v)^{-1}\right)}=\frac{3}{1}
$$

\subsection{Intensity correlation for non mode locked laser radiation}

Intuitively, one might think that the superposition of a large number of modes with stable amplitude but with random phases, i.e., not phase locked, would lead to an emission that is almost constant in intensity with possibly some small fluctuations. This was found to be absolutely not the case. Numerical calculations show, that even the free-running (not mode locked) multimode emission of a laser consists of a series of random intensity fluctuations with the shortest duration possible by the optical bandwidth (Figure 4). It corresponds to thermal or Gaussian light emission of the same bandwidth. For this situation, the intensity correlation can be exactly calculated. The result (Figure 5) shows that $G_{t h}^{2}(\tau)$ for this random emission changes from an initial value $G_{t h}^{2}(0)=2$ to
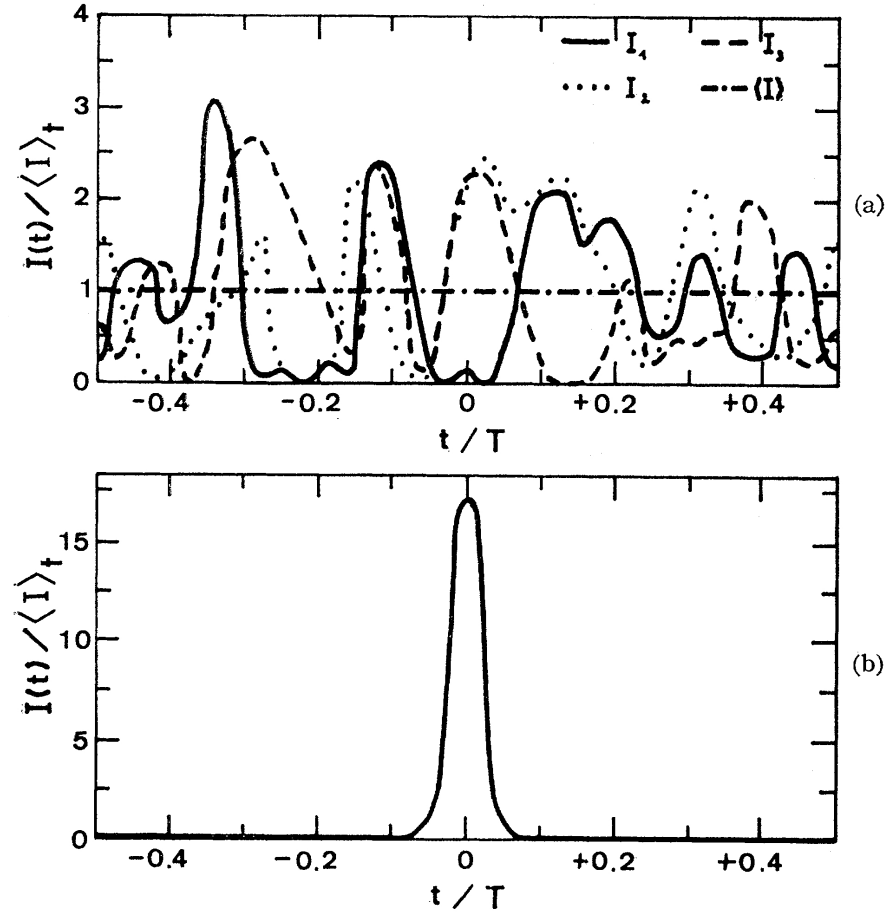

FIG. 4 Instantaneous intensity of a laser with 31 modes, having a Gaussian envelope, normalized to the mean intensity $\langle I\rangle_{t} . T$ is the repetition time. (a) Three examples with random phases between the modes, producing random intensity fluctuations. (b) Intensity of the same laser modes ideally mode-locked [10].

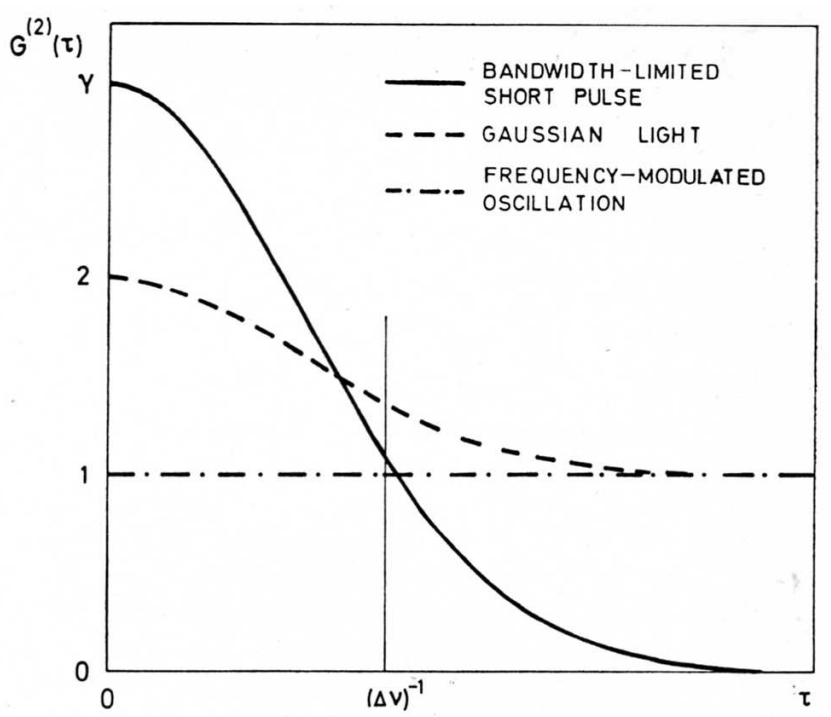

FIG. 5 Second order correlation function $G^{2}(\tau)$ for three types of light emission, all of the same oscillating bandwidth $(\Delta v)[7]$.

$G_{t h}^{2}\left(\tau \gg(\Delta v)^{-1}\right)=1$. The calculated peak to background ratios become thus for second harmonic generation

$$
C_{i d}^{S H}=\frac{G^{2}(0)}{G^{2}\left(\tau \gg(\Delta v)^{-1}\right)}=\frac{2}{1}
$$

and for two-photon absorption induced fluorescence

$$
C_{t h}^{T P A}=\frac{G^{2}(0)+2 \cdot G^{2}(0)}{G^{2}(0)+2 \cdot G^{2}\left(\tau \gg(\Delta v)^{-1}\right)}=\frac{1.5}{1} .
$$

The existence of these random intensity fluctuations for freerunning (non Q-switched) lasers was experimentally con- 
firmed by an autocorrelation measurement using the second harmonic approach with a highly controlled geometrical arrangement. Both, the total bandwidth and the exact theoretically predicted contrast could be confirmed [8].

From these results we conclude that the experimentally realized values for the reported mode locked lasers did not correspond to ideal mode locking. $\mathrm{SH}$ measurements prove the existence of isolated pulse, but they are not as short as theoretically possible; TPA measurements show the expected shortest possible events, however, the contrast ratio was far below the required value for isolated short pulses.

\section{IMPERFECTLY MODE LOCKED LASER EMISSION}

An entirely different approach to determine the pulse duration used as optical nonlinear process transient stimulated Raman scattering [9]. These experiments confirmed fully the time duration measured by the intensity correlation arrangement with second harmonic generation.

An open question remained however. What was the reason, that the emitted light pulses were not as short as they could have been? A numerical simulation of imperfectly modelocked laser emission and its effect on nonlinear optics could finally explain all the observed experimental results [10]. The assumption that the individual modes were not properly locked was simulated by random deviations of the phases of individual modes. The total emitted radiation can be described by

$$
I(t)=V(t) V^{*}(t)=\sum_{n, m} a_{n} a_{m} \exp \left\{i\left[(n-m) \Omega t+\varphi_{n}-\varphi_{m}\right]\right\},
$$

where $\Omega$ is the basic frequency of the laser resonator and $n$ and $m$ are mode orders, $a_{n}$ and $a_{m}$ are amplitudes and $\varphi_{n}$ and $\varphi_{m}$ are the relative phase angles. For ideal mode locking, $\varphi_{n}$ and $\varphi_{m}$ are all equal to zero. The corresponding result, a bandwidth limited short pulse, is shown in Figure 4(b). Examples for randomly distributed phases in a given interval are shown in Figure 6, together with the ensemble averaged intensity $\langle I\rangle$, which represents the distribution that will be measured. It is seen that the random distribution does not lead to longer time structures, however, they result in an increased background. The corresponding correlation functions $G_{i m p}^{2}(\tau)$ show in Figure 7 a background for $\tau \gg(\Delta v)^{-1}$, which is different from zero. This will reduce the contrast $C_{i m p}$ to values between $C_{i d}$ and $C_{t h}$, as observed in the respective experiments.

It was also shown (Figure 8), that only a systematic deviation of the phases between the locked modes at least quadratic with mode number, which corresponds to a frequency chirp, does increase the pulse duration. Obviously, the previously studied lasers produced such a chirp. Intensity correlation functions for different systematic phase distributions are shown in Figure 9: ideal mode locking (solid line), chirp (dashed line), FM-modulation (dash-dotted line). In the last case, the intensity is constant and the total bandwidth is generated by a periodic modulation (at the basic frequency $\Omega$ of the laser resonator) of the centre frequency of the laser emis-
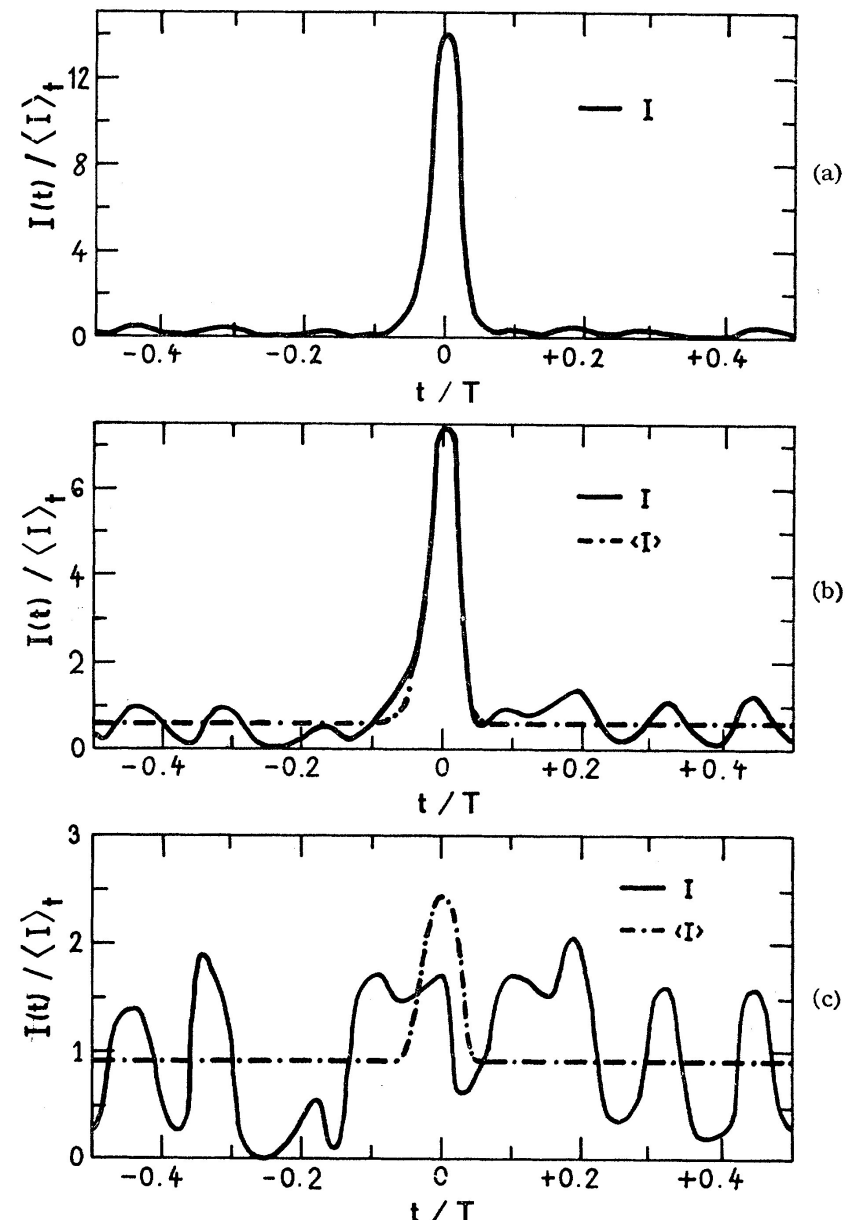

FIG. 6 Examples of the instantaneous intensity of a laser with 31 modes of amplitudes having a Gaussian envelope and phase angles $\varphi$ randomly distributed in different intervals [10]: (a) $-\frac{1}{4} \pi<\varphi<+\frac{1}{4} \pi$, (b) $-\frac{1}{2} \pi<\varphi<+\frac{1}{2} \pi$ and (c) $-\frac{3}{4} \pi<\varphi<$ $+\frac{3}{4} \pi$, with $T=\Omega^{-1}$ is the repetition time, $\langle I\rangle_{t}$ is the mean intensity and $\langle I\rangle$ the ensemble averaged intensity. Note that the scale of $I(t)$ differs in the three figures.

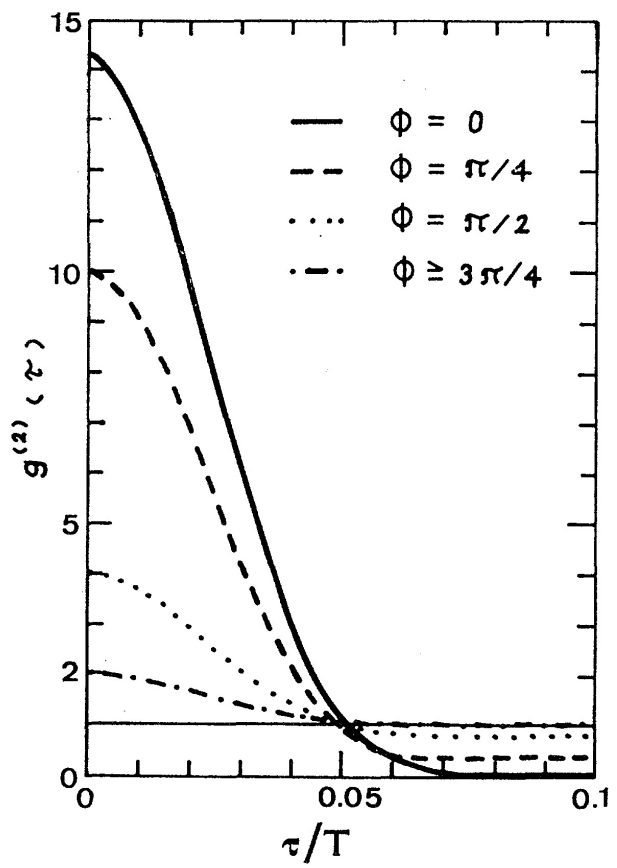

FIG. 7 Normalized intensity-correlation functions for different degrees of partial mode locking [10]. The phase angles are randomly distributed in the interval $-\Phi<\varphi<$ $+\Phi$. 


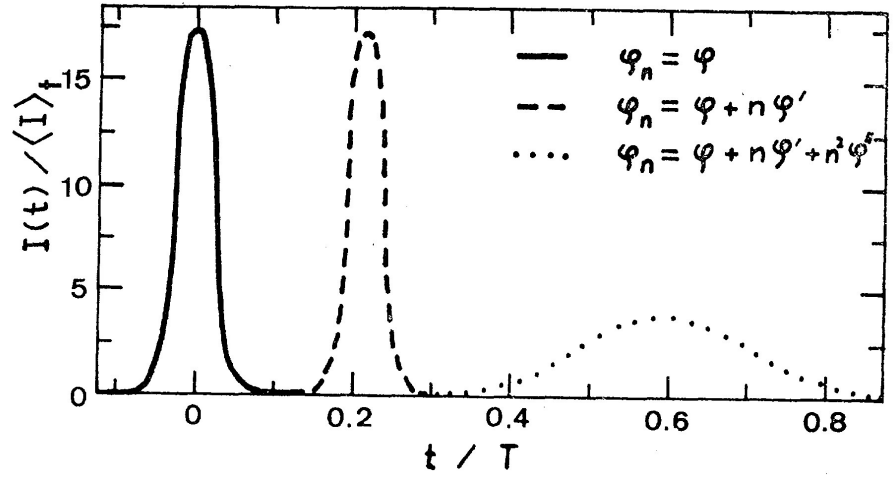

FIG. 8 Instantaneous intensity of a laser with fixed phase angles and a systematic deviation from ideal mode locking [10].

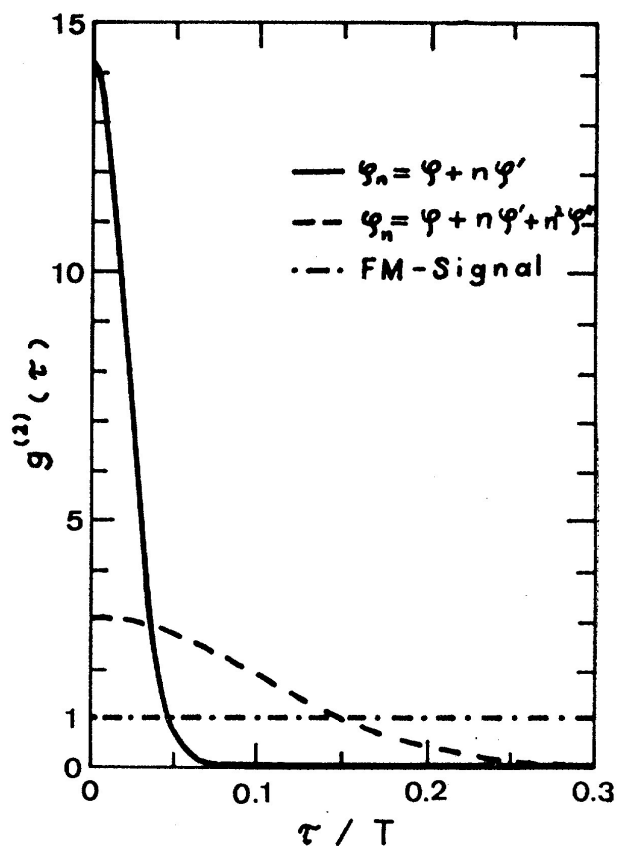

FIG. 9 Normalized intensity correlation functions for fixed phase angles with systematic deviation from ideal mode locking and the extreme case of an FM laser output [10].

sion (FM-modulation). The correlation function has a constant value equal to 1 .

\section{CONCLUSIONS}

The state of the art in the mid sixties with respect to evaluation of time duration of psec lasers has been reviewed. Due to the lack of ultra fast electronics, new methods had to be found to study these novel ultra-short mode locked laser pulses.In the search for new measuring methods we concentrated on nonlinear optics, the fastest coherent optical process. The discov- ery of the intensity correlation measurement based on second harmonic generation allowed to increase the time resolution by many orders of magnitude. With psec pulses, the shortest pulses at that time, the functioning of the method could be proven. A critical comparison with an alternative method, based on two photon induced fluorescence, is also presented. The interpretation of measured results led to the conclusion, that the psec pulses at that time were not yet as short as the total emitted bandwidth would have allowed. With a numerical analyses we showed that a chirp in the pulses was likely to be responsible for this observation.

It may be worthwhile to note, that it took several years until continuously running mode locked broadband lasers lead to significant progress in the field of ultra short pulses. However, the method for measuring their time duration remained essentially the same. The method holds even for fsec pulses, where only minor modifications of the instrumentation were necessary.

\section{References}

[1] A. J. DeMaria, D. A. Stetzer, and H. Haynau, "Self mode-locking of lasers with saturable absorbers" Appl. Phys. Lett. 8, 174 (1966).

[2] H. P. Weber, E. Mathieu, and K.P. Meyer, "Optical mixing with different relative polarizations of the beams" J. Appl. Phys. 37, 3584 (1966).

[3] H. P. Weber, "Method for pulsewidth measurements of ultrashort light pulses generated by phase-locked lasers using nonlinear optics" J. Appl. Phys. 38, 2231 (1967).

[4] H. P. Weber, "Generation and measurement of ultrashort light pulses" J. Appl. Phys. 39, 6041 (1968).

[5] J. A. Giordmaine, P. M. Rentzepis, S. L. Shapiro, and K. W. Wecht, "Two-photon excitation of fluorescence by picosecond light pulses" Appl. Phys. Lett. 11, 216 (1967).

[6] H. P. Weber, "Comments on the pulse width measurement with two photon excitation of fluorescence" Phys. Lett. 27A, 321 (1968).

[7] H. P. Weber, and R. Dändliker, “Intensity Interferometry by TwoPhoton Excitation of Fluorescence" IEEE J. Quantum Elect. QE-4, 1009 (1968).

[8] E. Mathieu, and H. J. Keller, "Intensity correlation functions of a non-Q-switched laser, measured by second-harmonic generation" J. Appl. Phys. 41, 1560 (1970).

[9] G. G. Bret, and H. P. Weber, "Transient stimulated Raman scattering in liquids and evaluation of picosecond pulse duration" IEEE J. Quantum Elect. QE-4, 807 (1968).

[10] A. A. Grütter, H. P. Weber, and R. Dändliker, "Imperfectly modelocked laser emission and its effects on nonlinear optics" Phys. Rev. 185, 629 (1969). 